\title{
Recurrent Low-Grade Fibromyxoid Sarcoma of the Neck: Report of a Case and Review of the Literature
}

\author{
Aikaterini Mastoraki ${ }^{1,2}$ - Theodoros Strigkos ${ }^{1}$ - Fotios-Panagiotis Tatakis ${ }^{1}$. \\ Angela Christophi ${ }^{1} \cdot$ Vassilios Smyrniotis $^{1}$
}

Received: 20 August 2014 / Accepted: 15 June 2015 /Published online: 30 June 2015

(C) Indian Association of Surgical Oncology 2015

\begin{abstract}
Low-grade fibromyxoid sarcoma (LGFMS) represents a rare, indolent, benign neoplasm that proliferates at various anatomical locations. Histologically, the tumor consists of bland, deceptively benign-appearing spindle cells in a whirling pattern, as well as myxoid and collagenized stroma. The current treatment includes surgical excision. The aim of this investigation was to analyze a rare clinical case of recurrent LGFMS of the neck. Relevant literature is briefly reviewed. An 81-year-old male was admitted with an enlarging painless nontender mass in the neck. The patient referred a past medical history of LGFMS. Magnetic Resonance Imaging (MRI) depicted a large, well-defined mass in the lower neck and supraclavicular area, extending till the left upper area of the anterior thoracic wall. Based on the imaging characteristics and the medical history, a wide surgical excision of a $5.6 \times 6.6 \times 3.3 \mathrm{~cm}$ area, in close proximity to the cervical vertebrae was performed. On microsopic examination, the tumor contained alternating fibrous and mixoid components, with mixed hypo- and hyper-cellular areas, minimal necrosis and negligible cellular pleomorphism or nuclear atypia. As LGFMS is a soft tissue malignancy, any recurrences are usually local; therefore treatment of choice is radical, wide margin surgical excision. Nevertheless, the ideal goal of large safety margin of healthy tissue might be unattainable if it requires resection of vital anatomic structures. Because LGFMS has a very low mitotic rate, neither
\end{abstract}

Aikaterini Mastoraki

dr_kamast@yahoo.gr

1 4th Department of Surgery, Athens University, Medical School, ATTIKON University Hospital, 1 Rimini str., 12462 Chaidari, Athens, Greece

22 Irodotou street, 15122 Maroussi, Athens, Greece chemotherapy nor radiotherapy is expected to have significant effect on long-term prognosis.

Keywords Low-grade fibromyxoid sarcoma $\cdot$ Clinical features $\cdot$ Diagnostic approach $\cdot$ Surgical management

\section{Introduction}

Soft tissue sarcomas (STS) are mesenchymal neoplasms that have a usually low-grade potential for malignancy and account for about $1 \%$ of all malignant tumors [1]. Among them, lowgrade fibromyxoid sarcoma (LGFMS) represents a rare, indolent, benign and distinctive sarcoma first reported by Evans in 1987 [2]. Most patients range in age from 25 to 46 years with an equal incidence in men and women [3]. LGFMS proliferates at various anatomical locations, especially in subcutaneous or superficial soft tissue on the proximal extremities, as a painless, slow-growing, non-tender, firm mass. It is commonly reported to derive from the deep soft tissues, particularly the thigh, but occasionally described to arise from the chest wall, axilla, inguinal region, shoulder, arm, forearm, neck, mediastinum and brain [4]. Local recurrence and metastasis, usually to the lungs, have been detected at variable but significant rates [5]. Typically, LGFMS appears as a well-described encapsulated mass, without necrosis or hemorrhage [3]. Histologically, the tumor consists of bland, deceptively benign-appearing spindle cells in a whirling pattern, as well as myxoid and collagenized stroma. The heterogeneous histological appearance makes the diagnosis challenging. In spite of its benign histopathologic features, LGFMS displays malignant behavior and presents a paradoxically aggressive tendency with a high percentage of recurrence and metastasis [4]. More recently, LGFMS was proved to carry the recurrent chromosomal translocation $\mathrm{t}(7 ; 16)(\mathrm{q} 33 ; \mathrm{p} 11)$ encoding a FUS-CREB3L2 fusion oncoprotein which is considered to be specific for this entity 
$[4,5]$. The current treatment includes surgical excision. The aim of this investigation was to analyze a rare clinical case of a recurrent LGFMS of the neck, which is atypical for this type of sarcoma. Relevant literature is briefly reviewed. Clinical features, histogenesis, morphological and immunohistochemical findings are discussed, while the role of current diagnostic and therapeutic management of this uncommon lesion is emphasized.

\section{Case Report}

An 81-year-old male was admitted in our hospital with an enlarging painless mass in the neck, without any complaints of dyspnea or dysphagia. Upon physical examination, we identified a firm, non-tender mass in the lower left area of the neck, immovable on deglutition, without any palpable lymph nodes. The patient referred a past medical history of a LGFMS in the same area of the neck that was excised in another hospital 2 years prior to this admission. On a thirdparty laboratory, advanced medical imaging was performed on the neck and thorax. The Magnetic Resonance Imaging (MRI) study depicted a large, well-defined mass in the lower neck and supraclavicular area, extending till the left upper area of the anterior thoracic wall without invading any ribs. On T1T2 weighted and fluid-sensitive images, the mass presented as soft tissue lesion, while no fat, calcification, or acute hemorrhage were identified. Enlarged lymph nodes were absent; nevertheless the lesion was pressing against and constricting the internal jugular vein. On contrast-enhanced MRI images, the entire mass displayed homogeneous enhancement, a rather uncommon characteristic for LGFMS. Lung metastases were not documented. Additional Computed Tomography (CT) scan of relevant areas provided similar findings (Fig. 1). All routine investigations yielded normal results. Based on both

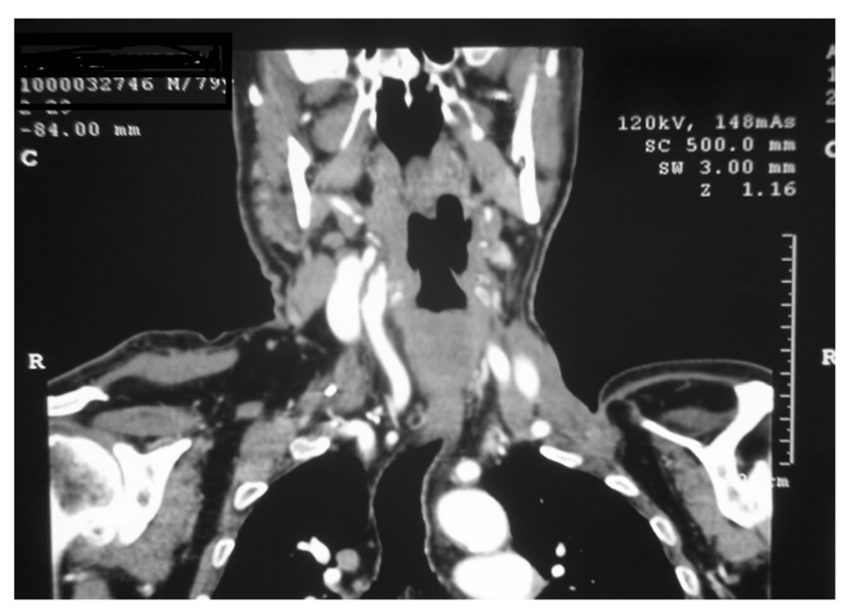

Fig. 1 CT depiction of a large, well-defined mass with homogenous enhancement in the lower neck and supraclavicular area, extending till the left upper area of the anterior thoracic wall
MRI and CT imaging characteristics as well as the medical history, the mass in question as a relapse of the previously diagnosed LGFMS was elucidated, and a wide surgical excision of a $5.6 \times 6.6 \times 3.3 \mathrm{~cm}$ area, in close proximity to the cervical vertebrae was performed. Along with the mass, invaded structures including a part of the sternocleidomastoid muscle and branches of the brachial plexus were excised, and the internal jugular vein was ligated. Post-operative course was uneventful. On microsopic examination, the tumor contained alternating fibrous and mixoid components, with mixed hypo- and hyper-cellular areas, minimal necrosis and negligible cellular pleomorphism or nuclear atypia (Fig. 2). Immunohistochemically, the tumor cells were focally positive for Smooth Muscle Actin (SMA) and calponin, and negative for HHF-35, caldesmon, desmin, S-100, CD34, CD57 and $\beta$-catenin. The Ki-67 proliferation index was approximately $1 \%$.

\section{Discussion}

LGFMS is an increasingly recognized subtype of STS. Since its first description, the histopathology and clinical behavior of the tumor have been adequately established in the medical literature. This rare type of mesenchymal sarcoma is commonly seen in the young and middle-aged adults, although it can also occur at the extremes of life. LGFMS is most prevalent in young patients and it can be found in the lower extremities and deep soft tissue, with even sex distribution [3]. Nonetheless, Folpe et al. demonstrate a male predominance in accordance with other smaller series. Its true incidence is unclear and the disease-specific mortality is totally obscure $[5,6]$. The mean tumor size ranges from 1.4 to $19 \mathrm{~cm}$, although the majority varies approximately from 8 to $10 \mathrm{~cm}$ [5]. Relevant previous

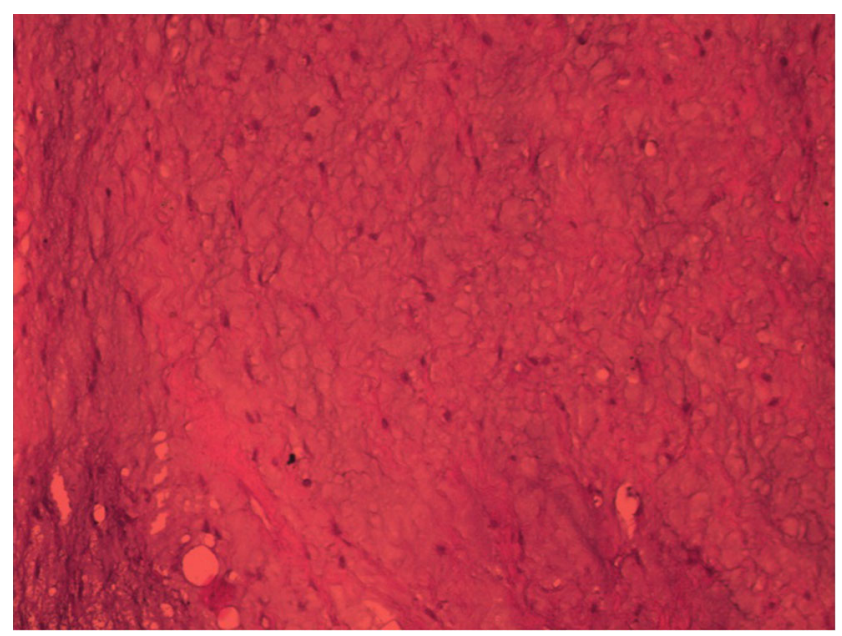

Fig. 2 Histological section of mesenchymal neoplasm showing loose and focally myxomatous stroma and sparse spindle cells (Hematoxylin - Eosin $\times 100$ ) 
investigations demonstrated that the neoplasm presents a substantial rate of local recurrence (33\%) and predominantly hematogeneous pulmonary metastasis $(5-58 \%)$ [7]. Herein we have the opportunity to describe an atypical recurrent LGFMS, which concerns an 81-year-old male. This specific clinical case is rare, as based on the previous reports and the sparse literature; it was found in the neck (atypical location) of an older male (low age incidence) in close proximity with the left carotid sheath and as a result the neoplasm not only constricts, but also causes internal jugular vein thrombosis (IJVT), a rare and serious clinical entity with a potentially fatal outcome [8].

Histologically, LGFMS typically presents contrasting fibrous and myxoid zones, with bland, benign-appearing spindle cells, exhibiting swirling or whorled growth patterns without significant necrosis. In general, hypercellular and hypocellular areas are noted, though hypercellular areas are more frequent in recurrent and metastatic tumors. Tumor cells generally present none or negligible nuclear and cellular pleomorphism. In addition, the observed mitotic rate is significantly low; at most 1 mitotic figure per 10 high-power fields (HPF) is commonly noticed, though that number may be higher in metastases $[7,8]$. Besides these typical microscopic findings, other structures have been recorded in the literature, such as osseous metaplasia, pericollagenous rosettes, cyst formation, and atypical growth patterns, including storiform or fascicular-herringbone [9]. Immunohistochemically, LGFMS is consistently strongly positive for vimentin, as a mesenchymal neoplasm, and typically negative for any lineage specific antibodies, such as SMA, $\beta$-catenin, desmin, S100, cytokeratin, CD34 and CD56 as well. The Ki-67 proliferation index is typically observed to be lower than $2 \%$, which is uncommon for malignancies, and is yet to be explained. In our specific case, the tumor presents with typical microscopic features, but is weakly positive for SMA and calponin, an uncommon characteristic and typically negative for other markers. Ki-67 proliferation rate is only $1 \%$. Therefore, the manuscript elaborates more pathological highlights, than clinical interest features as this rare nosologic entity appears with specific microscopic findings indicative of the diagnosis instead of certain clinical evidence.

The CT depicted hypodense and isodense areas probably correspond to the myxoid and fibrous stroma of LGFMS seen on histology respectively. The variable contrast enhancement pattern reflects different amount of fibrotic and myxoid tissue. MR findings are in absolute agreement with the pathological features. In general, fibrous tissue components appear hypointense on T1/T2-weighted images [3]. Additional slight enhancement is commonly encountered. Myxoid tissue components are usually hypointense on T1-weighted images and hyperintense on T2-weighted images. Fine-needle aspiration (FNA) biopsy for cytology is generally avoided, except to provide material for cytogenetic and molecular studies. LGFMS is characterized by a recurrent chromosomal translocation $\mathrm{t}(7 ; 16)(\mathrm{q} 33 ; \mathrm{p} 11)$ or $\mathrm{t}(11 ; 16)(\mathrm{p} 11 ; \mathrm{p} 11)$ encoding either FUSCREB3L2 or FUS-CREB3L1 fusion oncoprotein. As both translocations involve the FUS gene, FISH for FUS genetic rearrangement is an ideal technique for confirming the diagnosis $[5,10]$. Alternatively, reverse transcriptase polymerase chain reaction (RT-PCR) can also be used to detect either fusion product; such assays have only rarely been proposed to analyze formalin-fixed, paraffin-embedded neoplasm samples [11]. It is obvious that diagnosis is challenging; both long latency periods between diagnosis and recurrence, as well as slow tumor growth have been assessed, underlying the importance of a long-term clinical follow-up and imaging surveillance [3].

As LGFMS is a soft tissue malignancy, any recurrences are usually local; therefore treatment of choice is radical, wide margin surgical excision. In fact, the most important prognostic factors for the behaviour of this specific sarcoma are tumour size at diagnosis and negative surgical margins; patients with microscopically negative margins after aggressive surgical removal have a lower probability of relapse, and a longer period between recurrences [7]. However, the ideal goal of large safety margin of healthy tissue might be unattainable if it requires resection of vital anatomic structures that cannot be sacrificed, thus limiting the excision width. Radical excision, if possible, is also employed as the treatment of choice for all local recurrences and distant metastases (usually to the lungs). Because LGFMS has a very low mitotic rate, neither chemotherapy nor radiotherapy is expected to have significant effect on long-term prognosis [12]. Nevertheless, it has been suggested that adjuvant radiotherapy could delay recurrences and increase life expectancy. In our specific case, we treated the recurrence of the tumour with wide surgical excision, yet due to its proximity to the cervical vertebrae that could not be resected away, surgical margins were microscopically positive. Given the fact that LGFMS has the potential for very late recurrences and metastases, long-term follow-up is necessary.

\section{References}

1. Alevizopoulos A, Mygdalis V, Tyritzis S, Stravodimos K, Constantinides CA (2012) Low-grade fibromyxoid sarcoma of the renal pelvis: first report. Case Rep Nephrol Urol 2:87-91

2. Evans HL (1987) Low-grade fibromyxoid sarcoma. A report of two metastasizing neoplasms having a deceptively benign appearance. Am J Clin Pathol 88:615-619

3. Kim SK, Jee WH, Lee AW, Chung YG (2011) Haemorrhagic lowgrade fibromyxoid sarcoma: MR findings in two young women. $\mathrm{Br}$ J Radiol 84:146-150

4. Singh K, Singh S, Pal N, Sampley SK, Chhabra K (2012) Lowgrade fibromyxoid sarcoma of anterior abdominal wall. Indian $\mathrm{J}$ Surg 74:351-353

5. Rose B, Tamvakopoulos GS, Dulay K, Pollock R, Skinner J, Briggs T, Cannon S (2011) The clinical significance of the FUS-CREB3L2 translocation in low-grade fibromyxoid sarcoma. J Orthop Surg 6:15 
6. Folpe AL, Lane KL, Paull G, Weiss SW (2000) Low-grade fibromyxoid sarcoma and hyalinizing spindle cell tumor with giant rosettes: a clinicopathologic study of 73 cases supporting their identity and assessing the impact of high-grade areas. Am J Surg Pathol 24:1353-1360

7. Evans HL (2011) Low-grade fibromyxoid sarcoma: a clinicopathologic study of 33 cases with long-term follow-up. Am J Surg Pathol 35:1450

8. Abe Y, Hashimoto I, Nakanishi H (2012) Recurring facial lowgrade fibromyxoid sarcoma in an elderly patient: a case report. J Med Invest 59:266-269

9. He KF, Jia J, Zhao YF (2013) Low-grade fibromyxoid sarcoma with cystic appearance and osseous metaplasia in the cheek: a case report and review of the literature. J Oral Maxillofac Surg 71:11431150
10. Sedrak MP, Parker DC, Gardner JM (2014) Low-grade fibromyxoid sarcoma with nuclear pleomorphism arising in the subcutis of a child. J Cutan Pathol 41:134-138

11. Matsuyama A, Hisaoka M, Shimajiri S, Hayashi T, Imamura T, Ishida T, Fukunaga M, Fukuhara T, Minato H, Nakajima T, Yonezawa S, Kuroda M, Yamasaki F, Toyoshima S, Hashimoto H (2006) Molecular detection of FUS-CREB3L2 fusion transcripts in low-grade fibromyxoid sarcoma using formalin-fixed, paraffinembedded tissue specimens. Am J Surg Pathol 30:1077-1084

12. Delaney TF, Kepka L, Goldberg SI, Hornicek FJ, Gebhardt MC, Yoon SS, Springfield DS, Raskin KA, Harmon DC, Kirsch DG, Mankin HJ, Rosenberg AE, Nielsen GP, Suit HD (2007) Radiation therapy for control of soft-tissue sarcomas resected with positive margins. Int J Radiat Oncol Biol Phys 67:1460-1469 